\title{
STUDY OF SOME FACTORS ASSOCIATED WITH HIGH-RISK ASTHMA IN CHILDREN
}

Inass M. Taha *, Sahar M. Ali **, Ahmad Abdelsadek Mohammad ***, and Nany M.S. Nawar ****

*Internal Medicine Department, Faculty of Medicine, Taibah University, Saudi Arabia. **Microbiology and Immunology Department, Faculty of Medicine, Menufiya University, Egypt. ***Chest Department, Faculty of Medicine, Benha University, Egypt. ****Immunology Department, King Fahad Hospital (MSC biochemistery), Saudi Arabia.

\begin{abstract}
Background: Bronchial asthma is characterized by lower respiratory tract inflammation associated with bronchial hyper responsiveness with variable and reversible airflow obstruction. The majority of asthmatics are sensitized to at least one common allergen.
\end{abstract}

Aim of the study:

The aim of this study is to determine the association of high-risk asthma with allergy-related parameters (total serum IgE levels, serum levels of allergen- specific IgE, eosinophil count, eosinophil percentage ) and pulmonary functions in children.

Subjects and methods: 50 Children aged 8-15 years diagnosed with atopic asthma were enrolled in the study. Pulmonary function tests, total leukocyte count (TLC), eosinophil count, and eosinophil percentage were estimated. Total serum immunoglobulin E (IgE) levels and serum IgE levels specific to antigens from 1 to 9 allergens with class 1 or higher, namely, Dermatophagoides pteronyssinus (D. pteronyssinus), Dermatophagoides farina (D. farina ), cat dander , dog dander, cockroach, egg white, milk, Aspergillus fumigatus, and fish, were measured using UniCAP fluoroenzyme immunoassay (FEIA).

Results: This study includes 50 participants, 20 (40\%) belonged to the high-risk and $30(60 \%)$ to the low-risk groups. This study revealed no significant association in peak expiratory flow rate (PEFR, \% ) values between high-risk and low-risk asthma groups $(p>0.05)$. There was no significant association in forced expiratory volume in first second [ FEV1 (L)] values between high-risk and low-risk asthma groups $(\mathrm{p}>0.05)$, whereas there was significant association in FEF $25-75 \%$ (forced expiratory flow 25-75\%) values between high-risk and low-risk asthma groups $(\mathrm{p}<0.05)$. There was a significant association between total serum IgE level and high-risk asthma, but TLC, eosinophil count, and eosinophil percentage showed non significant association with high-risk asthma. Serum levels of IgE specific to D. pteronyssinus, D. farina, cat dander, and dog dander were significantly associated with high-risk asthma. The high-risk group had higher serum levels of $\operatorname{IgE}$ specific to D. pteronyssinus ( $\mathrm{p}<0.0001)$, D. farina $(\mathrm{p}<0.0001)$, cat dander $(\mathrm{p}<0.0001)$, and $\operatorname{dog}$ dander antigens $(\mathrm{p}<0.0001)$ compared with those in the low-risk group. There was no significant association between high-risk asthma and the serum levels of IgE specific to antigens from other allergens (including cockroach, egg white, and milk). Serum levels of IgE specific to Aspergillus fumigatus and fish were both negative (class level < 1) in both the high-risk group and the low-risk group.

\section{Conclusion}

Children with higher serum levels of IgE specific to D.pteronyssinus, D. farina, cat dander and dog dander antigens, and total serum IgE levels, and lower FEF25-75\% values belong to the high-risk asthma group. The characterization of risk factors has enabled us to identify high-risk asthma in children, leading to better treatment options.

Key words: HIGH-RISK ASTHMA, CHILDREN. 


\section{Introduction}

Bronchial asthma is characterized by lower respiratory tract inflammation associated with bronchial hyper responsiveness with variable and reversible airflow obstruction [1] Asthma is the most common chronic disease among children. According to the World Health Organization, approximately 300 million people currently have asthma and approximately 250,000 patients die each year [2]. Aside from its increasing prevalence, the severity of asthma also seems to be increasing in pediatric and adolescent patients, which is based on the observed increase in rates of consultations and visits into clinics, hospitals, and emergency departments [3] IgE is an antibody subclass implicated in airway inflammation and allergic reactions. High levels of IgE are associated with asthma in both adults and children. IgE may also play a role in modulating the severity of asthma because previous studies have found associations between high IgE levels and greater asthma severity, airway hyper responsiveness, and lower baseline lung function [4] Despite the importance of genetic factors in the development of asthma, environmental factors, especially indoor aeroallergens, are probably the primary determinants of asthma expression and are essential for understanding the etiology of asthma. The majority of asthmatics are sensitized to at least one common allergen [5].

\begin{abstract}
Aim of the study
The aim of this study is to determine the association of high-risk asthma with some allergy-related parameters (e.g., total serum IgE levels, serum levels of allergen-specific IgE, eosinophil count, eosinophil percentage) and pulmonary functions in children.
\end{abstract}

\section{Subjects and methods}

This study was carried out on patients with physician-diagnosed atopic asthma. The participants were 50 children (18 male and 32 female) with ages ranging from 6 to 15 years. Their parents helped in answering the questions fully. This study was carried between January 2012 and June 2012 at Madina National Hospital and King Fahad
Hospital, Kingdom of Saudi Arabia. Demographic data (i.e., age, gender, height, and weight) were obtained through interviews. All patients were also subjected to a clinical evaluation (i.e., asthma severity and asthma score). All subjects were submitted to the following:

1-Full history taking (parents helped in answering the questions fully) and clinical examination.

2- Radiological examination (plain Chest X ray posteroanterior and lateral views).

3 -Ventilatory function tests (spirometry) by using Spiroanalyzer ST-95, serial number 67011870, Fukuda Sangyo 2003. The best of three consecutive spirometry recordings were used. Measurements included assessments of PEFR\% (peak expiratory flow rate \%), FEV1 (forced expiratory volume in first second), and FEF $25-75 \%$ (forced expiratory flow, 25-75\%). 4- Routine laboratory investigations : Complete blood count (CBC), Total leukocytic count (TLC), absolute eosinophil count (AEC) and eosinophil percentage were made.

5-Serum levels of Total and allergen-specific $\mathrm{IgE}$ :Blood was drawn from all participants at the start of the study and allowed to clot for 1 hour in stable room temperature before centrifugation. Serum was pipetted and stored at $-70^{\circ} \mathrm{C}$ for further measurement of total serum IgE levels and serum IgE specific to antigens from the 9 allergens stated above. Serum was analyzed using the UniCAP fluoroenzyme immunoassay (FEIA) according to manufacturer's instructions (Phadia $A B$, Uppsala, Sweden). The lower limit of detection (LLOD) was $1 \mathrm{IU} / \mathrm{mL}$ for total $\mathrm{IgE}$ and $0.35 \mathrm{IU} / \mathrm{mL}$ for each of the allergenspecific IgEs; the upper limit of detection (ULOD) was $1,000 \mathrm{IU} / \mathrm{mL}$. For total $\mathrm{IgE}$ testing, no samples exceeded the ULOD of $50,000 \mathrm{IU} / \mathrm{mL}$. The CAP classes were scored as follows: $0.35-0.70 \mathrm{IU} / \mathrm{mL}$, class $1 ; 0.71-3.5$ $\mathrm{IU} / \mathrm{mL}$, class 2 ; 3.6-7.5 IU/mL, class 3 ; 7.6$17.5 \mathrm{IU} / \mathrm{mL}$, class $4 ; 17.6-50 \mathrm{IU} / \mathrm{mL}$, class 5 ; and $>50 \mathrm{IU} / \mathrm{mL}$, class 6 . For total $\mathrm{IgE}$, values $\geq 1 \mathrm{IU} / \mathrm{mL}$ were considered positive and for the specific $\mathrm{IgE}$, values $\geq 0.35 \mathrm{IU} / \mathrm{mL}$ were considered positive. 
In principality, The CAP System FEIA employs a type of "architecture" where by the allergen of interest is covalently bound to a hydrophilic carrier polymer, encased in a capsule, which catches all allergen- specific $\operatorname{IgE}$ in the sample. Allergen-specific $\operatorname{IgE}$ is detected directly with a combination of polyclonal and monoclonal anti- $\operatorname{IgE}(\mathrm{Fc})$ antibodies labeled with beta- galactosidase, generating fluorescence. The specific IgE antibody in the sample is connecting to the allergens in the immunoCAP after buffer solution has been washed away (Pre-wash), all not connected sample is washed away (sample wash ). Antibodies in the conjugate connects to the $\operatorname{IgE}$ during conjugate incubation, excess conjugate is washed away in the conjugate wash. Development solution is added and reacts with the conjugate connected to the ImmunoCAP, After development incubation stop solution is added to halt the process.
Children with atopic asthma were defined as patients diagnosed with asthma who had $\mathrm{IgE}$ specific to antigens from 1 to 9 allergens with class 1 or higher, namely, D. pteronyssinus, D. farina, cat and dog dander, cockroach, egg white, milk, Aspergillus fumigatus, and fish. High-risk asthma was defined as asthma requiring hospital admission or an emergency department visit within the year before enrollment in the study. All other patients with asthma were classified as having low-risk asthma.

Asthma severity was classified into four categories according to the GINA [2] guidelines and based on the level of symptoms, degree of airflow limitation, and pulmonary function variability (Table A). These were intermittent, mild persistent, moderate persistent, and severe persistent. Each asthma severity score ranges from 1 to 4 points.

6-Case definition

Table (A) Classification of asthma severity

\begin{tabular}{|c|c|c|c|c|}
\hline & \multicolumn{2}{|c|}{ Frequency of symptoms } & \multirow{2}{*}{$\begin{array}{c}\% \text { Predicted } \\
\text { FEV1/PEFR\% }\end{array}$} & \multirow{2}{*}{$\begin{array}{l}\text { Variability } \\
\text { PEFR\% }\end{array}$} \\
\hline & Day & Night & & \\
\hline Intermittent & $<1 /$ wk & $\leq 2 / \mathrm{m}$ & $\geq 80 \%$ & $<20 \%$ \\
\hline Mild persistent & $\begin{array}{l}\geq 1 / \text { wk } \\
<1 / \text { day }\end{array}$ & $2 / \mathrm{m}$ & $\geq 80 \%$ & $20-30 \%$ \\
\hline Moderate persistent & Daily & $\geq 1 /$ wk & $60-80 \%$ & $>\mathbf{3 0 \%}$ \\
\hline Sever persistent & Daily & Frequent & $\leq 60 \%$ & $>\mathbf{3 0 \%}$ \\
\hline
\end{tabular}

Classified according to the Global Initiative for Asthma guidelines [2]

Intermittent: 1 point

Mild persistent: 2 points

Moderate persistent: 3 points

Severe persistent: 4 points

The asthma symptoms score and possible total score ranged from 0 to 16 points (Table B). 
Table (B) Asthma symptom score

\begin{tabular}{l}
\hline Cough score (nighttime) \\
\hline = Absent \\
1 = Mild (cough was present but did not disturb sleep) \\
$2=$ Moderate (patient awoke once because of cough) \\
$3=$ Severe (patient awoke more than once because of cough) \\
$4=$ Extremely severe (patient experienced insomnia on most nights) \\
\hline Shortness of breath score (early in the morning) \\
\hline $0=$ Absent \\
$1=$ Mild (occasional shortness of breath that did not require \\
medication) \\
$2=$ Moderate (occasional shortness of breath that required \\
medication) \\
$3=$ Severe (frequent shortness of breath that required medication) \\
$4=$ Extremely severe (persistent shortness of breath that required \\
multiple doses of medication) \\
\hline Wheeze or dyspnea score (daytime) \\
\hline $0=$ Absent \\
$1=$ Mild (occasional wheezing) \\
$2=$ Moderate (occasional wheezing and dyspnea that did not disturb \\
normal activities) \\
$3=$ Severe (persistent wheezing and dyspnea that hampered normal \\
activities) \\
$4=$ Extremely severe (wheezing and dyspnea that left the patient \\
totally unable to perform normal activities) \\
\hline Cough score (day time) \\
$0=$ Absent \\
$1=$ Mild (occasional cough that did not disturb normal activities) \\
$2=$ Moderate (frequent cough that did not disturb normal \\
activities) \\
$3=$ Severe (frequent cough that disturbed normal activities) \\
$4=$ Extremely severe (persistent cough) \\
\hline
\end{tabular}

\section{Statistical methods:}

IBM SPSS statistics (V. 21.0, IBM Corp., USA, 2012) was used for data analysis. Data were expressed as mean \pm SD for quantitative parametric measures in addition to median percentiles for quantitative nonparametric measures and both number and percentage for categorized data.

\section{Discussion}

Asthma is a major public health problem that affects millions of children worldwide. In recent years, the frequency and severity of asthma have increased [6]. Despite the importance of genetic factors in the development of asthma, environmental factors, especially indoor aeroallergens, are probably the primary determinants of asthma expression and are essential for understanding the etiology of asthma. The majority of asthmatics are sensitized to at least one common allergen [5]. Allergic diseases represent a significant percentage of chronic pediatric illnesses. There are several reasons why it is important to identify the specific allergen sensitivity with the appropriate allergy diagnostic tests. Clinical history may not be sufficient to correctly determine the specific allergen(s) causing the 
symptoms, and this may lead to inappropriate treatment recommendations such as unnecessary elimination diets or allergen avoidance measures [7]. In our study the rate of high-risk asthma in children was high ( $40 \%)$. This is the reason why it is necessary to determine the risk factors for high-risk asthma and define the high-risk group more accurately. The classification based on severity is useful in making decisions regarding management during initial patient assessment. The term "asthma severity" indicates both the severity of the disease and a patient's responsiveness to treatment [2]. The demographic data of the studied subjects included in this study are illustrated in Table 1. The high-risk asthma group consisted of 20 patients, 6 males $(30 \%)$ and 14 females (70\%), with an average age of $9.7+1.8$ years, average weight of 35.4+9.8, and average BMI of $(18.5+1.7)$, whereas the low-risk asthma group consisted of 30 patients, 12 males $(40 \%)$ and 18 females $(60 \%)$, with an average age of $10.9+2.06$ years, average weight of $39.8+11.9$, and average BMI of $19.7+2.2$ $\mathrm{g} / \mathrm{m}^{2}$ This study revealed no significant association in PEFR (\%) values between high-risk and low-risk asthma groups $(57.4+14.8$ vs. $61.6+18.8, \mathrm{p}=>0.05)$. There was no significant association in FEV1 (L) values between high-risk and low-risk asthma groups $(34.8+5.59$ vs. $37.0+7.23, \mathrm{p}>$ 0.05), although there was significant association in FEF25-75\% values between high-risk and low-risk asthma groups $(32.6+7.86$ vs. $41.5+7.27, \mathrm{p}<0.05)$ as shown in Table 2.

These results were in agreement with those of Chieh et al. [8] who studied the association between serum levels of IgE and asthma severity among Mexican, Puerto Rican, and African American patients with asthma, overlying 1122 participants, of which $412(37 \%)$ and $710(63 \%)$ patients belonged to the high-risk and low risk groups, respectively. There was significant association between FEF25-75\% value and high-risk asthma. The FEF25-75\% values in the high-risk group were significantly lower than those in the low-risk group ( $48 \pm 20$ vs.
$53 \pm 20, \mathrm{p}<0.05)$. There was no significant association between high-risk asthma and PEFR (\%) and FEV1 values. This could be explained by the following: children at all levels of asthma severity can have relatively unimpaired FEV1 values when they are clinically stable. In children the distal airways are definitely affected, and normal FEV1 values are typically seen in many children with asthma due to increased peripheral airway resistance in the absence of or before significantly large airway involvement. Several studies have shown abnormalities in FEF25-75\% values in asthmatic children with normal FEV1 values [9]. Luigi et al. [10] found that reduced FEF25-75\% values in intermittent allergic asthma patients are significantly higher than the FEV1 and PEFR $(\%)$ values. Accordingly, only the FEF25-75\% values correlate with high-risk asthma in children. In our study, there was no significant association between TLC, eosinophil count, and eosinophil percentage in high-risk and low-risk groups. These levels were $7.2+1.9$ vs. $6.9+1.3, \mathrm{p}>0.05 ; 330+193$ vs. $253+69.7$, $\mathrm{p}>0.05 ; 4.07+1.29$ vs. $3.5+1.9, \mathrm{p}>0.05$, although there was significant association in total serum IgE levels between the high-risk group and the low-risk group $(1062+841 \mathrm{vs.}$ $101+60.4, \mathrm{p}<0.05$ ), respectively, ( Table $3)$.

These results are in agreement with Chieh et al. [8] who found that total serum IgE levels in the high-risk group were higher than those in the low-risk group $(786.56 \pm 1110.52$ vs. $596.32 \pm 862.87, \mathrm{p}<0.05)$, but no significant difference between high risk and low risk as regard eosinophil count ( high-risk $4.75 \pm 4.79$ vs. $4.77 \pm 3.80, p>0.05)$. A strong association between asthma and elevated total serum IgE levels has been reported in cross-sectional and prospective studies by Wever et al. [11] and Siroux et al. [4] who noted that total serum IgE level is positively associated with asthma-related hospitalization in a 12-month period before enrolment in the study. Their findings are consistent with our observations. Total serum IgE level is positively correlated with high-risk asthma. 
However, Gergen et al. [12] examined the association between total IgE levels and asthma in the National Health and Nutrition Examination Survey and noted that total IgE levels are associated with asthma only among persons who have positive results for at least 1 allergen-specific IgE. A few studies have examined the relationship between blood eosinophil count and asthma severity in children. These studies show a positive correlation between blood eosinophil counts and the severity of asthma symptoms [13].

A prospective study involving more than 1000 patients with asthma has shown that peripheral blood eosinophil count $>0.45 \times$ $10^{9}$ per liter is associated with a more than 7fold increase in the relative risk of asthmarelated death [14]. However, eosinophil counts were not related to asthma severity in a study in which asthma patients were monitored form childhood to adulthood [15]. This may be explained by the fact that blood eosinophils do not exactly reflect eosinophilic airway inflammation [16] and neutrophils, rather than eosinophils, may be the predominant cells in high-risk asthma [17].

The present study revealed that serum levels of IgE specific to D.pteronyssinus, D. farina, cat dander, and dog dander were significantly associated with high-risk asthma. The high-risk group had higher serum levels of $\operatorname{IgE}$ specific to $\mathrm{D}$. pteronyssinus $(6.18 \pm 3.24$ vs. $2.30 \pm 0.96, \mathrm{p}<$ $0.0001)$, D. farina $(3.53+1.93$ vs. $1.45+0.55$, , $\mathrm{p}<0.0001)$, cat dander ( $2.02 \pm 0.34$ vs. $0.62 \pm 0.10, \mathrm{p}<0.0001)$, and dog dander antigens $(1.41 \pm 0.28$ vs. $0.35 \pm 0.11,, \mathrm{p}<$ $0.0001)$ compared with those in the low-risk group. There was no significant association between high-risk asthma and the serum levels of IgE specific to antigens from other allergens (including cockroach, egg white, and milk). Serum levels of IgE specific to Aspergillus fumigatus and fish were both negative (class level $<1$ ) in both the highrisk group and the low-risk group (Table 4 and figure (1)). In both the high-risk group and the low-risk group, the number of patients sensitized to Aspergillus fumigatus or fish allergen was zero. Hence, the use of
IgE specific to Aspergillus fumigatus or fish as a risk factor for high-risk asthma is not useful in clinical practice.

Similar results were found by Williams et al. [18] who revealed a positive association between asthma and serum-specific IgE for cat dander, dog dander, and dust mites (D. pteronyssinus, D.farina). Nearly similar results were obtained by Emara et al [19] who concluded that the majority of asthmatic patients were sensitive to dust mites $(60 \%)$, followed by mixed grass pollens (30\%), Penicillium notatum (25\%), Cockroach (20\%) resp

ectively . Also Sporik et al. [20] found that sensitization to cat or dog dander allergens is strongly associated with bronchial hyperreactivity. Early allergen sensitization is associated with an increased risk of persistent asthma. More importantly, sensitization to indoor aeroallergens is the factor that shows the strongest association with asthma. Early exposure to dust mite allergens is a significant factor in the development of asthma. Sensitization to house dust mite and cat dander allergens is also associated with frequent hospital visits in older children [21]. However, other studies have produced conflicting results. For instance, Carlsten $\mathbf{C}$ et al. [22] have found that sensitization to dog allergens is not a predominant risk factor for asthma. Also, a major study in the northeastern region of the United States of America has shown the importance of cockroach allergen exposure and sensitization in asthma severity among inner-city children [23]. Exposure to indoor allergens causes asthma and allergy, but this effect may depend on the dose and type of allergen, as well as the underlying genetic susceptibility of the child [21].

\section{Conclusion}

Children with higher serum levels of $\operatorname{IgE}$ specific to D.pteronyssinus, D. farina, cat dander and dog dander antigens, and total serum IgE levels, and lower FEF25-75\% values belong to the high-risk asthma group. The characterization of risk factors has enabled us to identify high-risk asthma in children, leading to better treatment options. 


\section{References}

1-Beeh KM, Ksoll M, Buhl R(2000): Elevation of total serum immunoglobulin $\mathrm{E}$ is associated with asthma in nonallergic individuals. Eur Respir J .,16:609-14.

2-Global Strategy for Asthma Management and Prevention, Global Initiative for Asthma (GINA), 2008.

3-Chipps BE, Szefler SJ.(2007): Demographic and clinical characteristics of children and adolescents with severe or difficult-to-treat asthma.J. Allergy Clin Immunol .119:1156-63.

4-Siroux V, Oryszczyn M-P, Paty E, Kauffmann F, Pison C, Vervolet D.(2003): Relationships of allergic sensitization, total immunoglobulin $\mathrm{E}$ and blood eosinophils to asthma severity in children of the EGEA Study. Clin Exp Allergy ;33:74651.

5- Giavina-Bianchi P, Fidalgo S, Duarte A.J.S.(1996): Hipersensibilidade dos pacientes com asma e rinite na cidade de São Paulo. Arch Argent Inmunol. Clín.;27:12.

6- Francisca XM Rego, Pedro-Giavnia Bianchi, Jorge Kalil, L. Karla Arruda and Myrthes Toledo Barros(2011): The hammock: a reservoir of allergens clinics (Sao Paulo). Arch Argent Inmunol. Clín ;66(7):1199-202.

7-Linda Cox ( 2011): Overview of SerologicalSpecific IgE Antibody Testing in Children. Springer Science, S 11882-011-0226-3.

8-Chieh-Han Cheng1, Shyh-Dar Shyur1, Li-Hsin Huang1, Yu-Hsuan Kao1, Wei-Te Lei1, Chia-Yi Lo1, Kuo- Hsi Lee1, Chen-Kuan Chen1, and Ling-Chun Liu Asian Pac(2010):

J. Allergy Immunol;28:250-5.

9-Gelfand W, Kraft M (2009): The importance and features of the distal airways in children and adults. J. Allergy Clin. Immunol;124:S84-7.

10-Luigi DB, Emanuel DT, Federica DB, Fabrizio DT.(2007): FEF75 in asthma management. Eur. Ann. Allergy Clin. Immunol. ;39(10):333-6.

11-Wever-Hess J, Kaowenberg JM, Duiverman EJ, Hermans J, Wever AMJ.(2000):Risk factors for exacerbations and hospital admissions in asthma of early childhood. Pedia Pulmo.;29:2506.

12-Gergen PJ, Arbes SJ, Calatroni A, Mitchell HE, Zeldin DC.(2009):Total IgE levels and asthma prevalence in the US population: results from the National Health and Nutrition
Examination Survey 2005-2006. J Allergy Clin Immunol;124:447-53.

13-Ulrik CS, Frederiksen J.(1995): Mortality and markers of risk of asthma death among 1075 outpatients with asthma. Chest;108:10-5.

14-Ulrik CS.(1995):Peripheral eosinophil counts as a marker of disease activity in intrinsic and extrinsic asthma. Clin. Exp. Allergy :25:820-7.

15-Grol MH, Postma DS, Vonk JM .(1999): Risk factors from childhood to adulthood for bronchial responsiveness at age 32-42 yr. Am. J. Respir. Crit. Care Med.;160:150-6.

16-Pizzichini E, Pizzichini MM, Efthimiadis A, Dolovich J, Hargreave FE(1997) :Measuring airway inflammation in asthma: eosinophils and eosinophilic cationic protein in induced sputum compared with peripheral blood. J. Allergy Clin. Immunol.;99:539-44.

17-Jatakanon A, Uasuf C, Maziak W(1999):Neutrophilic inflammation in severe persistent asthma. Am. J. Respir. Crit. Care Med. ;160:1532-9.

18-William AH, Smith JT ,Hudgens EE, Rhoney $\mathrm{S}$, Ozkaynak H , Hamilton RG and Gallagher JE.(2011): Allergens in household dust and serological indicators of atopy and sensitization in Detroit children with history-based evidence of asthma. J. Asthma ;48(7):674-84.

19-Emara M.M., Mansour H.A., Shehata M.T. and Zakia Abu-Zahab(2012): Outcome of Sublingual Immunotherapy with Multiple Allergens in Asthmatic Patients with and without Allergic Rhinitis.; Life Science Journal ; 9(3) :817-827.

20-Sporik R, Ingram JM, Price W (1995): Association of asthma with serum IgE and skintest reactivity to allergens among children living at high altitude: tickling the dragon's breath. Am J Respir Crit Care Med ;151:1388-92.

21-Arshad SH(2010): Does exposure to indoor allergens contribute to the development of asthma and allergy. Curr. Allergy Asthma Rep. ;10(1):49-55.

22-Carlsten C, Dimich-ward H, Becker AB (2010): Indoor allergen exposure, sensitization, and development of asthma in a high-risk birth cohort. Pediatr. Allergy Immunol. DOI:10.1111/j.1399- 3038.2010.01021.x.

23-Rosenstreich DL, Eggleston P, Kattan M (1997): The role of cockroach allergy and exposure to cockroach allergen in causing morbidity among inner-city children with asthma. N. Engl. J. Med. May 8;336(19):1356-63. 


\section{Results}

Table (1) Characteristics of the patients of high-risk and low-risk asthma

\begin{tabular}{|c|c|c|}
\hline Parameter & $\begin{array}{l}\text { High-risk } \\
\text { number }(\%)\end{array}$ & $\begin{array}{l}\text { Low-risk } \\
\text { number (\%) }\end{array}$ \\
\hline $\begin{array}{l}\text { Male } \\
\text { Female }\end{array}$ & $\begin{array}{l}6(30 \%) \\
14(70 \%)\end{array}$ & $\begin{array}{l}12(40 \%) \\
18(60 \%)\end{array}$ \\
\hline Age (mean+SD)/year & $9.7+1.8$ & $10.9+2.06$ \\
\hline Average weight/kg & $35.4+9.8$ & $39.8+11.9$ \\
\hline Average body mass index (BMI) kg/m² & $18.5+1.7$ & $19.7+2.2$ \\
\hline
\end{tabular}

Table (2) Association between pulmonary function and high-risk asthma

\begin{tabular}{|c|c|c|c|c|}
\hline Parameter & Group & Mean (SD) & t-test & $\mathbf{P}$ \\
\hline \multirow[t]{2}{*}{ PEFR (\%) } & High-risk ( 20 ) & $57.4+14.8$ & \multirow[b]{2}{*}{0.84} & \multirow[b]{2}{*}{$>0.05$} \\
\hline & Low-risk ( 30) & $61.6+18.8$ & & \\
\hline \multirow[t]{2}{*}{ FEV1 (L) } & High-risk ( 20 ) & $34.8+5.59$ & \multirow[b]{2}{*}{1.15} & \multirow[b]{2}{*}{$>0.05$} \\
\hline & Low-risk ( 30 ) & $37.0+7.23$ & & \\
\hline \multirow[t]{2}{*}{ FEF25-75\% } & High-risk ( $20 \quad$ ) & $32.6+7.86$ & \multirow[b]{2}{*}{4.11} & \multirow[b]{2}{*}{$<0.05$} \\
\hline & Low-risk ( 30 ) & $41.5+7.27$ & & \\
\hline
\end{tabular}

Table (3) Association between high-risk asthma and TLC, eosinophil count, eosinophil \%, and total serum IgE levels

\begin{tabular}{|l|l|l|l|l|}
\hline Parameter & $\begin{array}{l}\text { High risk } \\
(\text { mean+SD) }\end{array}$ & $\begin{array}{l}\text { Low risk } \\
(\text { mean+SD })\end{array}$ & t-test & P \\
\hline Total leukocyte count (TLC)/deciliter & $7.2+1.9 \times 1000$ & $6.9+1.3 \times 1000$ & 0.67 & $>0.05$ \\
\hline Eosinophil count/deciliter & $330+193$ & $253+69.7$ & 2.006 & $>0.05$ \\
\hline Eosinophil \% & $4.07+1.29$ & $3.5+1.9$ & 1.17 & $>0.05$ \\
\hline IgE ( IU/mL) & $1062+841$ & $101+60.4$ & 6.27 & $<0.05$ \\
\hline
\end{tabular}


Table (4) Association between high-risk asthma and serum levels of allergen-specific IgE (D1 = D. pteronyssinus, D2 = D. farina, E1 = cat dander, E5= dog dander, F 3= fish, M3 = Aspergillus fumigatus, I6 = cockroach, F1 = egg white and F2 = milk)

\begin{tabular}{|l|l|l|l|l|l|}
\hline Specific Ig E & $\begin{array}{l}\text { High risk } \\
\text { asthma }\end{array}$ & Low risk asthma & t-test & P & Significance \\
\hline D. pteronyssinus (D1) & $6.18 \pm 3.24$ & $2.30 \pm 0.96$ & 6.19 & $<0.0001$ & HS \\
\hline D. farinae (D2 ) & $3.53 \pm 1.93$ & $1.45 \pm 0.55$ & 5.59 & $<0.0001$ & HS \\
\hline Cat dander (E1) & $2.02 \pm 0.34$ & $0.62 \pm 0.10$ & 21.31 & $<0.0001$ & HS \\
\hline Dog dander ( E5) & $1.41 \pm 0.28$ & $0.35 \pm 0.11$ & 18.75 & $<0.0001$ & HS \\
\hline Cockroch ( I 6 ) & & & & & NS \\
\hline Egg white (F1) & $0.42 \pm 0.16$ & $0.40 . \pm 0.02$ & 0.68 & 0.499 & NS \\
\hline Milk (F2) & $1.20 \pm 0.59$ & $1.14 \pm 0.50$ & 0.39 & 0.700 & NS \\
\hline
\end{tabular}

Serum levels of IgE specific to Aspergillus fumigatus and fish were both negative (class level < 1) in both the high-risk group and the low-risk group.

Fig. ( 1 ): Comparison between high and low risk asthma group as regards the serum levels of allergen-specific IgE ( D1 , D2 ,E1,E5,I6, F1 and F2)

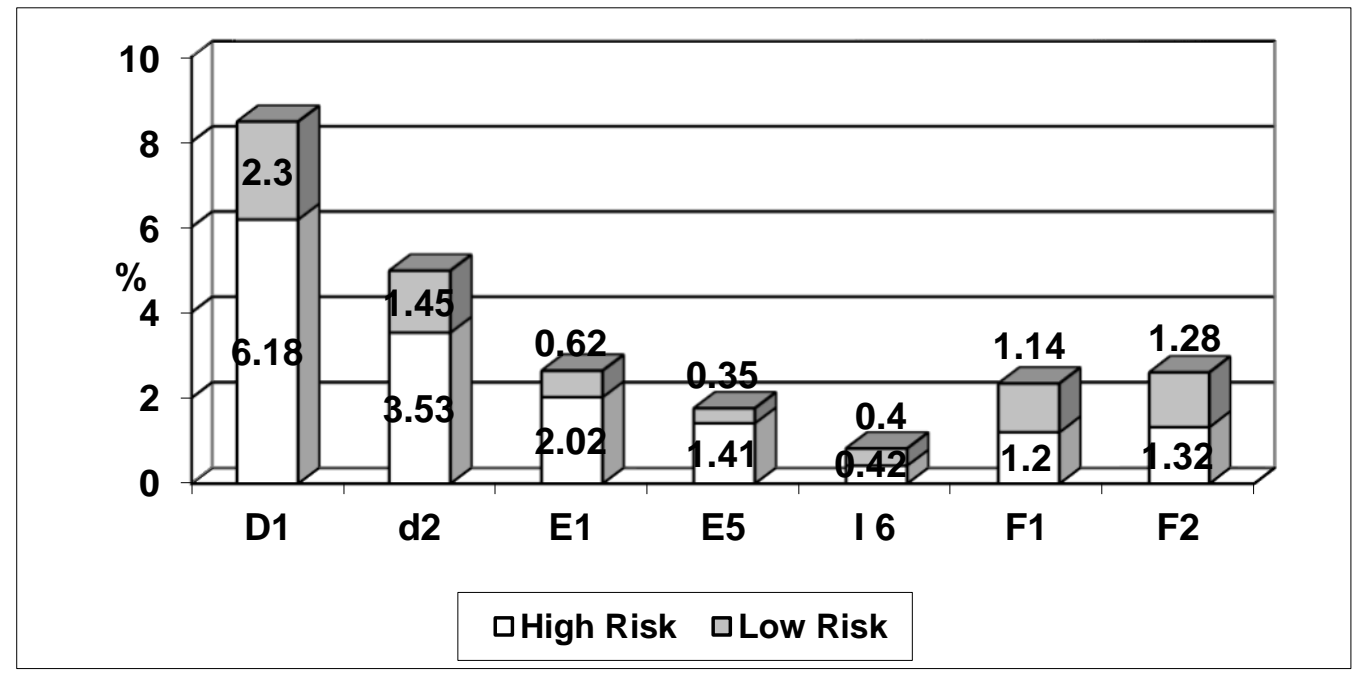

